\title{
Treatment-resistant depression: problematic illness or a problem in our approach?
}

\author{
Gin S. Malhi, Pritha Das, Zola Mannie and Lauren Irwin
}

\section{Summary}

Treatment-resistant depression is widely defined as nonresponse to two 'adequate' courses of treatment. However, the definitions of treatment and depression are inconsistent reflecting gaps in our understanding. We argue that a failure to respond is often the result of administering inappropriate treatment, which occurs principally because of paradigm failure.

\section{Declaration of interest}

None.

\section{Keywords}

Depressive disorders; antidepressants; bipolar affective disorders; treatment

\section{Copyright and usage}

(c) The Royal College of Psychiatrists 2018
Gin S. Malhi (pictured) manages patients with mood disorders in the Royal North Shore Hospital CADE Clinic in Sydney, Australia. Pritha Das is a research scientist with an interest in the neuroimaging of mood disorders, based at the CADE Clinic at Royal North Shore Hospital. Zola Mannie is a senior research fellow with an interest in the neurobiological aspects of mood disorders, based at the CADE Clinic at Royal North Shore Hospital. Lauren Irwin is a research assistant in the CADE Clinic. She has an interest in the psychological aspects of mood disorders.

'The source of resistance is the assurance that the older paradigm will ultimately solve all its problems, that nature can be shoved into the box the paradigm provides.'

Thomas Kuhn (The Structure of Scientific Revolutions, 1962)

There is ongoing debate over how to define treatment-resistant depression (TRD) and when to pursue alternative or experimental treatment strategies, although most define TRD as non-response to two 'adequate' courses of treatment. ${ }^{1-3}$ While discussions of what qualifies as resistance and advice regarding which steps to take when dealing with a patient who is non-responsive are valuable, they often overlook two fundamental factors: our definitions of the diagnosis of depression and its treatment. It is apparent that the burden of depression continues to rise despite the increasing use of antidepressants, ${ }^{4}$ which suggests that there is a flaw in our understanding of the diagnosis and treatment of depression. We posit that, in practice, a failure to respond is often the result of having administered wholly inappropriate treatment; that which is unsuitable because of a paradigm failure. We therefore argue that the validity of our diagnostic system and the efficacy of our treatments have to be re-examined if we are to have an impact on treatment resistance in the context of depression.

\section{Failures of our diagnostic paradigm}

\section{Misdiagnosis}

The problem of TRD begins with diagnosis. To begin with, failures of our diagnostic paradigm creates many avenues for misdiagnosis. First, diagnostic difficulties stem from the fact that illnesses are longitudinal but that diagnostic systems operate cross-sectionally. Within the category of mood disorders specifically, it is clear that there are some diagnoses that serve as precursors to others, and diagnoses often change over time or following reappraisal. ${ }^{5}$ For instance, recurrent depressive episodes that mark the emergence of bipolar disorder are understandably regarded as major depression until a manic episode appears. But, if a manic episode is yet to occur in the course of the illness, and patients with bipolar disorder are managed as if they have major (unipolar) depression, antidepressants alone are likely to be ineffective and may even exacerbate the illness.

Second, there is a great deal of uncertainty inherent in a diagnostic system that depends on symptom observation and self-report, and which has substantial overlap in diagnostic criteria across disorders. ${ }^{6}$ Where disorders have been delineated from others without evidence to support which symptoms are pathognomonic of the disorder, errors in diagnosis are commonplace. This is the case with bipolar II disorder, which is not only difficult to distinguish from other bipolar spectrum disorders and unipolar depression, but is often confused with borderline personality disorder. ${ }^{7}$ Consequently, those with borderline personality disorder, who are usually best suited to psychotherapy, are instead prescribed medications commonly used to treat bipolar disorder, to which they are predictably non-responsive, and then wrongly regarded as being treatment resistant.

Third, some diagnoses that warrant distinction are no longer being captured adequately within psychiatric taxonomy (DSM-5, ICD-10). ${ }^{8,9}$ A good example of this phenomenon is mixed states. The DSM- 5 criteria for the 'mixed features specifier' excludes symptoms of distractibility, irritability and psychomotor agitation, which have been shown to be key features of those experiencing mixed states. ${ }^{10}$ This means that many cases of mixed depression are not diagnosed as such, and those that are identified are assigned the one allencompassing label. This results in those with mixed depression being treated incorrectly or inadequately, and here too antidepressants can exacerbate the symptoms of mixed depression, ${ }^{11}$ producing treatment-induced syndromes and putative treatment resistance.

Fourth, the set of diagnostic categories provided in these taxonomies is limited, and so the presentations of real-world patients with depression, that often do not align perfectly with the specified diagnostic criteria, are 'made to fit' into an available diagnosis (as the alternative may mean receiving no treatment at all). As a result, it is not uncommon for patients to end up being treated for a disorder that they do not quite have, with treatments that do not have proven efficacy for their specific symptomatology. It is thus not surprising that they fail to respond to treatment. 


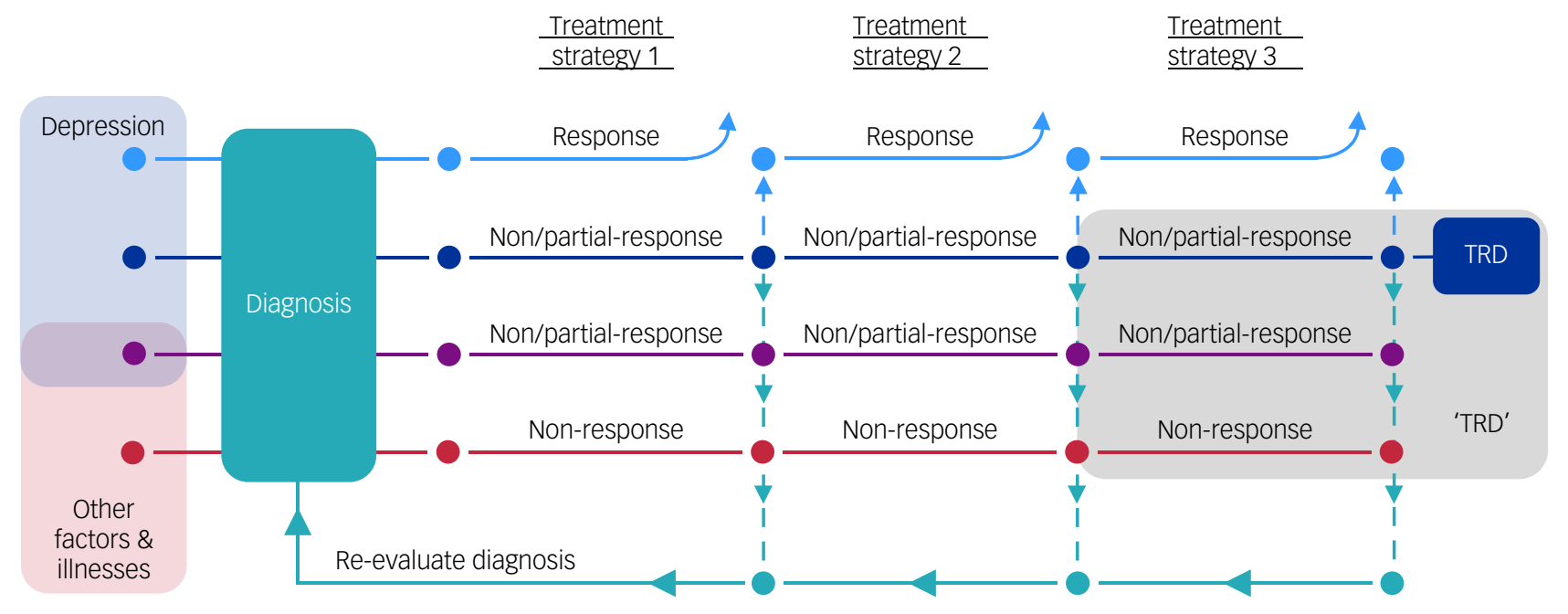

Fig. 1 An algorithmic approach to the diagnosis and treatment of depression.

The problem with treatment-resistant depression (TRD) lies at the outset where the diagnosis of depression overlaps with syndromes that can appear to be depression but are in fact either very different illnesses or are caused by factors that cannot be resolved by antidepressants alone (red). In these cases (some of which overlap with depression (purple)), partial- or non-response is to be expected. Those that have depression (blue) may also not respond to the very first treatment, or indeed the second or third treatment, but eventually, as shown in STAR* $D_{13}$, are likely to improve and are therefore responders.

Current definitions of 'TRD' identify 'treatment resistance' by trialling successive therapies. The most common definition requires a failure to respond to two adequate treatment trials of an antidepressant, though, in practice, treatments can include combinations of medications and augmentation strategies. The figure shows how the current definition of 'TRD' (grey) is arrived at through various treatment pathways and therefore fails to differentiate the many different causes of treatment resistance.

It also shows that within (overlapping) 'TRD' there is a kernel of actual treatment-resistant depression (dark blue) - in which the correctly diagnosed depressive illness is not responsive to currently available treatment strategies (including ECT). This kernel partly consists of clinical presentations for which antidepressant treatments have not been tested or shown to be effective, thus there should be no expectation that such a patient should respond. However, this kernel also contains patients with a clinical presentation that typically responds to antidepressant treatments, and this latter population can be considered to truly be treatment resistant.

\section{Diagnostic heterogeneity}

The risk of misdiagnosis is concerning, but even those accurately diagnosed with depression are susceptible to TRD that arises from paradigmatic failures, due to the lack of specificity of our diagnostic system. The diagnosis of depression is predicated on categorical diagnostic manuals, where a certain number of symptoms are required to be present for a particular duration of time in order to diagnose the disorder. The specific symptoms that 'define' a disorder have been drawn from clinical experience and epidemiological research that identifies which symptoms co-occur. This means that the underlying pathophysiology of the disorder is not considered when making a diagnosis (largely because, for depression and most psychiatric illnesses, this is not known). While the clinical picture of a patient is important, the lack of a reliable, objective indicator of depression has resulted in a heterogeneous patient population.

The extent of this heterogeneity can be illustrated by considering the mathematical combinations of symptoms. DSM- $5{ }^{10}$ requires five out of a set of nine symptoms, at least one of these being from two 'primary' symptoms, to be present for a diagnosis of depression to be made. If we consider patients who have five symptoms, more than a hundred presentations are possible, and this number doubles if we consider patients with six or more symptoms. For ICD-10, ${ }^{11}$ where four symptoms from a set of ten must be present, at least one from three 'primary' symptoms, the possibilities are even more numerous. Furthermore, in both of these sets of criteria there are dichotomous symptoms, such that a person with increased appetite, over-sleeping and psychomotor retardation fulfills the same symptoms as someone with no appetite, who is barely sleeping and experiencing psychomotor agitation; and this again increases the number of possible presentations that are nevertheless considered to be suffering from the same illness - namely, depression.

To add to this already broad set, there are several other 'depressive' symptoms that may also be present and colour the presentation of the illness: psychotic features, anxious distress, manic symptoms (i.e. mixed states), catatonia or melancholic features (DSM-5 also considers 'atypical' features). Furthermore, some patients experience seasonal variation, others experience depression in the peri-partum period and, whilst many have recurrent episodes, there are those that only experience a single episode in their whole life. Age of onset and hereditary loading are also highly variable between patients, and episode duration not only varies from patient to patient but can often change during the course of the illness as it progresses with age. Further still, depression is highly comorbid with anxiety, substance use and personality disorders. In sum, the combinations of symptoms and patterns that come under the label of 'depression' is myriad, and this needs to be taken into account when considering the treatment options available.

\section{The failure of our treatment paradigm}

Not surprisingly, the problems that concern diagnosis extend to the treatment domain. One of the idiosyncrasies of research is that clinical trials examining the efficacy of antidepressants have generally involved patients who have depression with 'pristine' symptom profiles that do not accurately reflect the heterogeneity of real-world presentations. ${ }^{12}$ Researchers and clinicians make the assumption that the positive treatment effects seen in these research populations are generalisable to all depressive presentations. However, STAR ${ }^{\star} \mathrm{D}$ and STEP-BD tested this assumption and revealed that, in realworld settings, our treatments are not as effective as portrayed in clinical trials. ${ }^{13,14}$ Collectively, findings from research to date suggest that 'antidepressants' are effective for a specific, homogeneous subset of depression. Therefore, labelling those who fail to respond to these medications as treatment resistant is disingenuous, and embarking on strategies such as switching and augmentation with medications that have also not been tested on heterogeneous populations is unlikely to benefit the patient. 


\section{Where to from here?}

There is thus one fundamental area that contributes greatly to putative TRD: poor diagnosis, which inevitably leads to poor treatment. Within these domains, responsibility falls to both clinicians and researchers to improve patient outcomes.

For researchers, it is imperative that efficacy studies are conducted on specific phenotypes to more precisely evaluate the conditions under which medications and psychotherapies are effective. This is necessary not only for identified subtypes of depression (for example, mixed, melancholic, psychotic) but also for groups delimited by characteristics such as early/late onset, hereditary loading and episode number and duration. It is also important to consider homogeneous symptom profiles and how these respond to medications and therapies. For example, patients with depression characterised by excessive sleep, fatigue, and a lack of interest in their usual activities are likely to respond differently to treatment than those diagnosed with depression based on feelings of hopelessness, intense guilt and persistent suicidal ideation. Furthermore, the effects of treatments on specific symptoms, not just overall scores, on scales of depression should also be considered. For example, research suggests that the benefit of atypical antipsychotics for depression is mostly because of improvements in sleep, ${ }^{15}$ and therefore sedative medications with less harmful side-effect profiles may be better alternatives for adjunctive treatment in cases where poor sleep is a key concern. Importantly, we know that a granular approach to profiling the clinical symptoms of depression can facilitate better treatment outcomes, as specific tests of psychotic and melancholic depression have revealed greater responsivity to electroconvulsive therapy. It is not unreasonable to expect that further differentiation in the efficacy of treatments can be achieved by specific examination of symptom profiles.

Research must also continue to test the validity of DSM diagnoses and diagnostic criteria. The fact is that our current diagnostic system is not perfect, and it is imperative that we work to improve it if we want to improve patient outcomes. The advent of modern technologies means that now more than ever we can test the biological validity of diagnoses. As such, research should not only consider studying the effects of medications on homogeneous symptom subgroups, but also examine whether such subgroups differ in neurocircuitry, genetic profiles and biomarkers, with the aim of identifying distinct phenotypes that are likely to respond differentially to treatment.

Perhaps the most difficult road ahead is that for clinicians (Fig. 1). Clinicians must be cognisant of the importance of identifying subtypes and acknowledge that antidepressants are not equally effective across the varied presentations of depression. Where research supports the use of antidepressants for a patient's symptomatology, they should be employed, but where there is uncertainty of the benefit that an antidepressant may have, clinicians should discuss this with the patient and exercise great caution in prescribing a medication that may instead confer an additional burden by producing considerable side-effects. ${ }^{16}$ Furthermore, the diagnosis needs to be re-evaluated when a medication fails, so as to allow for careful consideration of any new information that may explain the individual's purported resistance to treatment.

\section{Conclusions}

Finally, but perhaps foremost, the definition of TRD needs to be revised with an emphasis on diagnosis rather than treatment. The current definition of treatment resistance determined by treatment failure is tautological, and ignores the many reasons why a patient may not respond to a particular intervention. By defining TRD in this way, we are cementing the idea that it is an exceptional form of depression and not questioning whether the patient may have been misdiagnosed or may have a subtype of depression for which the benefit of antidepressants has not been demonstrated. This encourages increasingly risky treatment interventions that are likely to have negative effects on patients, and fails to encourage the development of novel therapeutics for specific subsets of patients that are non-responsive to current treatments. To defeat depression we do not always need to fight more aggressively, sometimes we simply need to alter our approach.

Gin S. Malhi (D), Professor, Discipline of Psychiatry, University of Sydney and Professor of Psychiatry, Royal North Shore Hospital CADE Clinic, Australia; Pritha Das, Research Scientist, Royal North Shore Hospital, Australia; Zola Mannie, Senior Research Fellow, Royal North Shore Hospital, Australia; Lauren Irwin (iD), Research Assistant, Royal North Shore Hospital, Australia

Correspondence: Gin S. Malhi, Discipline of Psychiatry, Sydney Medical School, University of Sydney, Sydney, NSW 2065, Australia. Email: gin.malhi@sydney.edu.au

First received 27 May 2018, final revision 25 Sep 2018, accepted 18 Oct 2018

\section{References}

1 Anderson IM. We all know what we mean by treatment-resistant depression don't we? Br J Psychiatry 2018; 212: 259-61.

2 Malhi GS, Byrow Y. Is treatment-resistant depression a useful concept? Evid Based Ment Health 2016; 19: 1-3.

3 McAllister-Williams R, Christmas D, Cleare A, Currie A, Gledhill J, Insole L, et al. Multiple-therapy-resistant major depressive disorder: a clinically important concept. Br J Psychiatry 2018; 212: 274-8.

4 Jorm AF, Patten SB, Brugha TS, Mojtabai R. Has increased provision of treatment reduced the prevalence of common mental disorders? Review of the evidence from four countries. World Psychiatry 2017; 16: 90-9.

5 Ruggero CJ, Carlson GA, Kotov R, Bromet EJ. Ten-year diagnostic consistency of bipolar disorder in a first-admission sample. Bipolar disorders 2010; 12: 21-31.

6 Silberman EK, Weiss KJ. Treatment-resistant depression or misdiagnosed anxiety disorder? J Nerv Ment Dis 2016; 204: 67.

7 Bassett D, Mulder R, Outhred T, Hamilton A, Morris G, Das P, et al. Defining disorders with permeable borders: you say bipolar. I say borderline! Bipolar Disord 2017; 19: 320-3.

8 American Psychiatric Association. Diagnostic and Statistical Manual of Mental Disorders, 5th edn (DSM-5). APA, 2013.

9 World Health Organization. The ICD-10 Classification of Mental and Behavioural Disorders: Clinical Descriptions and Diagnostic Guidelines. World Health Organization, 1992

10 Malhi GS, Berk M, Morris G, Hamilton A, Outhred T, Das P, et al. Mixed mood: the not so united states? Bipolar Disord 2017; 19: 242-5.

11 Faedda GL, Marangoni $\mathrm{C}$. What is the role of conventional antidepressants in the treatment of major depressive episodes with Mixed Features Specifier? CNS Spectr 2017; 22: 120-5.

12 Ghaemi SN. Why antidepressants are not antidepressants: STEP-BD, STAR* D, and the return of neurotic depression. Bipolar Disord 2008; 10: 957-68.

13 Rush AJ, Trivedi MH, Wisniewski SR, Nierenberg AA, Stewart JW, Warden D, et al. Acute and longer-term outcomes in depressed outpatients requiring one or several treatment steps: a STAR* D report. Focus 2008; 6: 128-42.

14 Sachs GS, Nierenberg AA, Calabrese JR, Marangell LB, Wisniewski SR, Gyulai L, et al. Effectiveness of adjunctive antidepressant treatment for bipolar depression. N Engl J Med 2007; 356: 1711-22.

15 Bauer M, El-Khalili N, Datto C, Szamosi J, Eriksson H. A pooled analysis of two randomised, placebo-controlled studies of extended release quetiapine fumarate adjunctive to antidepressant therapy in patients with major depressive disorder. J Affect Disord 2010; 127: 19-30.

16 Ghaemi SN. Toward a Hippocratic psychopharmacology. Can J Psychiatry 2008; 53: 189-96. 